\title{
Non-termination of sickness behavior as precipitating factor for mental disorders
}

\author{
Margaretha Viljoen and Annie Panzer \\ Department of Physiology, PO Box 2034, Pretoria 0001, South Africa
}

\section{Summary}

Sickness behavior can be defined as a combination of coordinated behavioral and physiological changes that develop in response to any condition that elicits pro-inflammatory activity. It is an adaptational homeostasis initiated by the influence of pro-inflammatory cytokines on central nervous system neurohormonal functioning. This paper introduces the concept of non-termination of sickness behavior as a potential threat to mental health. In view of the similarities between the behavioral symptoms, the neuroendocrine and the cytokine profiles of sickness behavior and that of a number of mental disorders it is hypothesized that the inappropriate continuation of sickness behavior, (i.e., non-termination), after recovery from the initial disease, could form the basis for mental disturbances. This would be particularly relevant in individuals with alterations in stress vulnerability (altered activation threshold and impaired negative feedback), which may occur due to the combination of genetic disposition and priming by early life

experiences.

\section{Background}

Sickness behavior can be defined as a combination of coordinated behavioral and physiological changes that develop in response to infection, inflammatory conditions, physical trauma or even negative emotions that initiate the inflammatory response [1]. The symptoms of sickness behavior can include any combination of the following: fever, fatigue, hyperalgesia, somnolence, anhedonia, loss of appetite, loss of interest in social and sexual interaction, decreased concentration and other cognitive abilities, decreased locomotor and body-care activities, as well as feelings of depression, hopelessness, irritability, anxiety, worthlessness, guilt and the reorganization of perceptions and coping strategies [2], [3] and [4]. Sickness behavior has been described as a central motivational state with selection of the appropriate strategy in response to the eliciting disease, in other words, a reorganization of behavior and other coping activities [1], [5] and [6]. There is ample evidence to show that, if any other factor is perceived as more threatening to the survival and well-being of the individual its motivational state may take precedence over the sickness motivational state, and behavior would be aimed at counteracting and coping with the new threat [1], [5] and [6]. Sickness behavior can thus be seen as a state of adapted homeostasis characterized by reorganization of coping responses and influenced by a perception-determined motivational state. It is important that the term sickness behavior should not be confused with the term illness behavior, an expression used to describe the way patients behave when stressed with illness - a personality and culture dependent phenomenon [7].

Although there are still gaps in our knowledge, the mechanisms underlying sickness behavior are generally well documented [2], [3], [6], [8], [9] and [10], and a heuristic presentation of sickness behavior in psychoneuroimmunological context is presented in Fig. 1. The process can be summarized by saying that infectious and inflammatory conditions, as well as most other forms of tissue injury, can stimulate the production of pro-inflammatory cytokines and that these peripherally produced cytokines can upregulate the basal cerebral pro-inflammatory cytokine production and release [9]. The peripherally produced cytokines can also change the basal neurohormonal activity and set points of the neuromodulatory systems like the CRH/HPA-axis (corticotropin-releasing hormone/ hypothalamo- 
pituitary-adrenocortical-axis), the CNA/SAM-axis (central noradrenergic/sympatho-adrenomedullaryaxis) and the serotonergic system [11] and [12] which, in turn, may lead to further upregulation of cerebral cytokine production [9]. This forms the basis for the new adaptational mind-body homeostasis (Fig. 1), i.e., sickness behavior, consisting of altered neurohormonal functioning, and adaptations in behavior, immunological activity and metabolic and motor responses. The symptoms of sickness behavior are intended to aid in the recovery from whatever condition initiated the increase in peripheral cytokine production. The adaptational value of the neurological, behavioral, metabolic, motor and immunological symptoms of sickness behavior covers a range of physiological and psychological advantages, including prevention of the spread of infections, optimizing the defense of the body against infection, injury and neoplastic growth, as well as reverting energy consumption to the processes of healing and recovery [13] and [14].

The major cytokines involved in the initiation of sickness behavior are T cell-derived Il-2 and the mononuclear cell-derived Il-6, Il-1 and TNF- $\alpha$, with Il-1 seen as the prototypical pro-inflammatory cytokine [6], [8] and [10]. Usually the cytokine profile and therefore the adaptive new homeostasis will revert back to normal upon recovery from the infection, the inflammatory condition or the physical trauma. There are, however, situations where the return to normal may be impeded. Cortisol resistance is probably the one major factor that can contribute to a delay in the return to normal homeostasis long after recovery from the initiating condition (discussed in more detail later).

It is intriguing that many of the symptoms of sickness behavior correspond to those seen in a number of mental disorders. These symptoms are largely the reflection of regional cytokine-induced neuroendocrine changes that include disturbances in the CRH/HPA-axis, the CNA/SAM-axis and the central serotonergic and dopaminergic systems [8] and [15]. With some exceptions the cytokine-induced changes show a strong resemblance to stress-induced changes [16] and disturbances of these same systems are also found in a variety of mental disorders. Another feature common to sickness behavior, as well as to several mental disorders, is the increase in pro-inflammatory cytokine activity. Mental disorders in which abnormal levels of pro-inflammatory cytokines have been reported include mood disorders [17], psychotic disorders [12] and [18], delirium, dementia and related cognitive disorders [19], [20] and [21], obsessive-compulsive disorders, and anxiety disorders [22]. There is abundant proof, not only from research on sickness behavior, but also from human cytokine therapy and experimental work that cytokines can cause neurobehavioral disturbances ranging from mild constitutional symptoms (e.g., fatigue, chills, headaches, fever) to serious behavioral problems such as major depressive moods, hallucinations, disorientation, delirium and psychosis [12] and [23].

In view of the similarities between sickness behavior and a number of mental disorders it is hypothesized that continuation of sickness behavior, (i.e., non-termination), after recovery from the initial disease, could form the basis for mental disturbances. This would be particularly relevant in individuals with alterations in stress vulnerability resulting from changes in the basal activity of the main neurohormonal stress axes or the immune system, or with abnormalities in the mechanisms that contribute to the appropriate termination of the neuroimmunological activity associated with sickness behavior. It is feasible to suspect that such predisposing conditions may occur as a result of the combination of genetic disposition and priming by early life experiences and that the type of disturbance likely to occur could be determined by these two predisposing factors. In view of the cross-sensitization between stressors [24] and the fact that psychological stress can lead to an increase in pro-inflammatory cytokine activity [24] it speaks for itself that psychological stress or a secondary inflammatory or infectious condition, even if subclinical, which coincides with sickness behavior due to another cause, could contribute to the behavioral disturbances and even exacerbate the condition with dire consequences for mental health. 


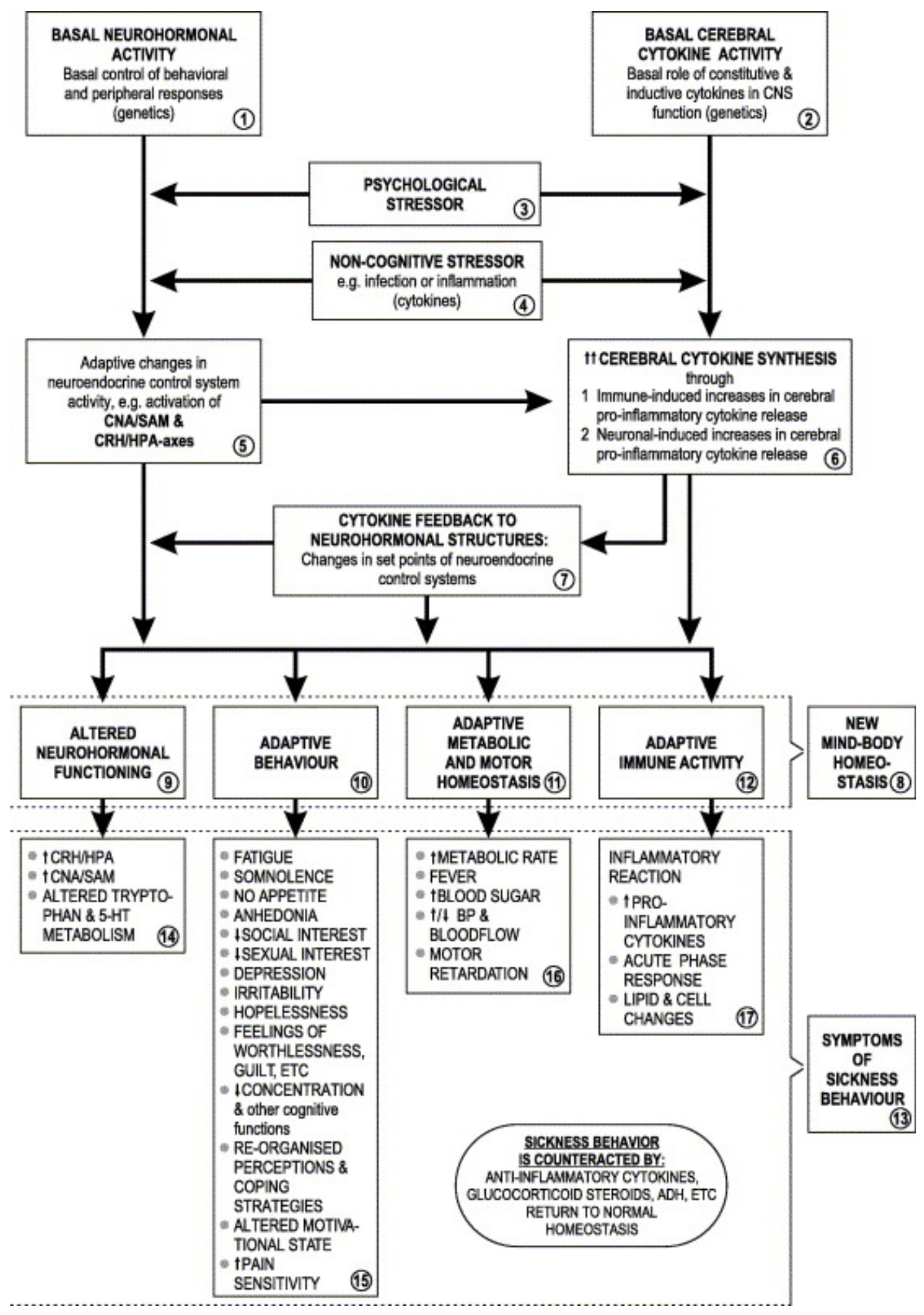

Figure 1. Sickness behavior in psychoneuroimmunological perspective. (1) and (2) represent the basal neurohormonal and basal immunological activity of the brain. These basal activities can be stimulated both by psychological stressors (3) and, as the case of infections, trauma and inflammatory conditions, by peripherally derived cytokines (4). The increase in basal neurological activity (5) would induce further cerebral cytokine production. Cerebral cytokine production is thus increased (6) as a result of peripheral immune activity as well as the increased neuronal activity. The marked increase in cerebral cytokine activity (6) can in turn feedback to the neurohormonal systems to bring about changes in their set points (7). The altered cerebral neurohormonal and cytokine activity will lead to a new adaptive mind-body homeostasis (8) intended to help cope with the stressor. Components of the new adaptive homeostasis include altered neurohormonal functioning (9), adaptive behavioral (10), adaptive metabolic and motor function (11) and adaptive immunological activity (12). The symptoms specific for the adaptive homeostasis of sickness behavior (13) are shown in (14), (15), (16) and (17). 


\section{Predisposition by early life experiences}

In this section, it is shown that the basal activity of neurohormonal systems can be primed to a lower activation threshold and suppressed negative feedback, with the implication for potential inappropriate continuation or non-termination of the sickness behavior homeostasis. Because of its central role in sickness behavior and in the stress response, predisposing of the central CRH/HPA-axis by early life experiences will be the main focus. However, there is also ample evidence for predisposing of the central noradrenergic, serotonergic and other systems [25].

Sensitive mothering and other optimal rearing experiences generally favour moderate stress responsiveness in adult life by increasing negative cortisol feedback to the hippocampus, and by decreasing the stress responsiveness of the hypothalamus, the pituitary, the amygdala, the BNST (bed nucleus of the stria terminalis) and the brain stem [26], [27], [28], [29] and [30]. In contrast, negative early life experiences can presensitize the individual to high stress vulnerability. Severe abuse is not necessarily needed for stress sensitization, as relatively common factors such as emotional neglect, poor infant-parent bonding, a cold or detached family environment, and periods of maternal separation can all alter the basic neuroendocrine-behavioral responsiveness and as such predispose to the development of mental and physical abnormalities in adult life [26].

It is known that stress-induced glucocorticoid secretion has opposing effects on different brain regions. CRH systems in the central nucleus of the amygdala and the BNST, in contrast to that of the paraventricular nucleus, increase their CRH expression in response to high levels of glucocorticoids and are able to stimulate the expression in other brain regions. Their dysregulation is strongly linked to low HPA-axis negative feedback and to conditions such as excessive shyness and fear in children, the development of anticipatory anxiety, melancholic depression and self-administration of psychotropic drugs [31]. Predisposing to stress vulnerability of the HPA-axis is mediated through mechanisms such as (a) increased forebrain noradrenergic activity that activates the HPA system and inhibits the GABA mechanisms [27], (b) increased CRH gene expression in the paraventricular nucleus [32], (c) decreased activity of the GABA and central benzodiazepine systems [32], and (d) serotonin-dependent changes in hippocampal cortisol receptor expression [33]. Mechanisms through which early experiences can potentiate high adult stress vulnerability through effects on the brain stem and amygdala, include (a) higher CRH-induced noradrenaline secretion (increased CRH receptor expression in the locus coeruleus, increased CRH mRNA in the central nucleus of the amygdala and decreased central benzodiazepine receptor expression in the amygdala and locus coeruleus), (b) increased CRH gene expression in the amygdala, altered noradrenergic and serotonergic pathways and suppression of the GABA-ergic/central benzodiazepine systems' activity involved in regulation of central CRH and noradrenergic activity [32], and (c) increased $\mathrm{CRH}$ transmission in the raphe nuclei, which alters serotonin activity and receptor expression in adult life [34]. CRH pathways innervate the dorsal raphe nucleus and it is known that adverse early life experiences can lead to CRH-induced augmentation of serotonin-dependent behavior. Although the interacting pathways between the main stress axes and serotonergic system are not always clear it is known that neonatal stress can cause desensitization of the 5-HT1A receptor-mediated autoinhibition, decrease sensitivity to 5-HT1A mediated inhibition of forebrain serotonin-release, decrease the response to alpha-1-adrenergic excitatory input and increase vulnerability to psychiatric illness in adulthood [35].

Predisposing to high stress vulnerability starts before birth and maternal stress has in utero influences on the offspring. Indications are that in utero exposure to increased glucocorticosteroids (e.g., maternal stress or therapeutic interventions) leads to HPA presensitization that is linked to premature psychopathologies in aging [36]. This together with evidence that CRH neurons are involved in target recognition and synaptic organization [37] may have serious implication for abnormal CRH activity during the gestational stages. Psychological stress is not the only factor that may change future stress responsiveness. Repetitive 
pain during neonatal intensive medical care, especially in premature infants, has been shown to alter neurological developments such as basal autonomic responses and arousal state [38]. In addition, infectious and inflammatory conditions during early life may also change CRH/HPA-responsiveness in adult life [39]. This is in agreement with the fact that immunological events can produce a CNS stress response similar to that initiated by psychological stressors. The reverse is also true, i.e., that early rearing experiences and social interactions may have long-, as well as short-term effects on the immune system [40].

There can be no doubt that stress-induced alterations in the CRH system can occur due to early life experiences and that these can persist throughout adult life. Hyperactivity of the CRH system is present in depression and in anxiety disorders, PTSD (posttraumatic stress disorder), Tourette's syndrome, obsessive-compulsive disorders, during fearful behavior with high frontal brain electrical activity, and in suicide victims. These alterations of the $\mathrm{CRH}$ system may at least partially result from the influence of early childhood experiences [41]. In adult women with a history of childhood abuse, multiple regression analyses showed childhood trauma, in addition to stressful periods in adulthood, to be the best predictor of high neurohormonal reactivity to stress tests or pharmacological challenges. These abused women also showed high CSF CRH levels, structural hippocampal changes and high levels of pro-inflammatory cytokines [42]. The examples given above, point predominantly to a predisposition to hyperactivity of the CRH/HPA system. However, hypoactivity may also develop. The mechanisms underlying a predisposition to hypoactivity are still largely unknown but certain indications are slowly emerging. It has for instance been reported that long-term desensitization of the HPA-axis may occur due to a single exposure to an extremely severe stressor. This appears to be mediated through paraventricular nucleus gene transcription and can be induced by emotional as well as by immunological events [43]. Evidence also exists that a blunted HPA-response may develop as a result of early painful experiences or chronic pain [44]. We are, however, still far from understanding the cumulative influences that will eventually determine stress responsiveness in adult life. The effect of intra-uterine predisposing to abnormal HPAreactivity, for instance, seems to depend on many factors, including birth weight and the gestational age at which the subject is born and it is suggested that, depending on such factors, fetal programming of the HPA axis may result in either hyper- or hypoactivation [45]. Whatever the mechanisms, all indications support the association between early life priming to altered activation threshold and impaired negative feedback on the one hand, and prolonged activation of the stress response on the other.

For sickness behavior, which constitutes a normal adaptational homeostasis, to progress to a mental disorder, i.e., inappropriate continuation of sickness behavior, there would have to be a failure in the mechanisms involved in the termination once the individual has recovered from the initiating onslaught. The next section will deal with this likelihood.

\section{Non-termination of sickness behavior}

Several factors are involved in the appropriate termination of sickness behavior, including negative feedback by cortisol on HPA-axis activity, downregulation of pro-inflammatory cytokine production due to high activity of the CNA/SAM and the CRH/HPA-axes [46] and [47], and control of cytokine production and effects by glucocorticoids. Cortisol, through its negative feedback on the hippocampus, anterior pituitary and hypothalamus [48], as well as its inhibition of the production of pro-inflammatory cytokines [47], can have a direct as well as an indirect suppressive effect on its own hypersecretion and on sickness behavior in general. The effects of glucocorticoids on cytokine production are mediated (a) by downregulation of pro-inflammatory cytokine synthesis and release through inhibition of transcriptional and post-transcriptional expression of the Il-1 $\beta$ gene and decreasing the stability of the Il$1 \beta$ mRNA, (b) by decreasing the ratio of type I Il-1 receptors, i.e., the functional receptors, to type II Il-1 receptors, i.e., the decoy receptors, and (c) by suppressing the conversion of pro-Il-1 $\beta$ to its biological active form through inhibition of the Il-1 $\beta$ converting enzyme [49]. Several other substances are also 
involved in the control of pro-inflammatory activity and their effects on behavior, including the antiinflammatory cytokines, antidiuretic hormone (ADH), $\alpha$-melanocyte stimulating hormone, and even certain members of the interleukin-1 family such as the Il-1 receptor antagonist [3] and [49]. However, the two most important factors in the control of pro-inflammatory activity is firstly that the excessive peripheral production of pro-inflammatory cytokines ceases to exist once the infection has cleared up, and secondly that the increased cortisol secretion and high SAM activity, which form part of the sickness behavior profile, cause a shift in cytokine production away from pro-inflammatory cytokines, in favour of anti-inflammatory cytokines [46] and [47].

Several factors can contribute to the continuation of sickness behavior, including basal hyperactivity of the stress systems. Predisposing to a hyperactive CRH/HPA-axis was previously discussed, with the major mechanisms being the downregulation of hippocampal and upregulation of amygdalar glucocorticosteroid receptors and therefore a weaker negative feedback [50]. In view of the detrimental effects of the CRH/HPA-axis [51], it is clear how such a predisposition can lead to the continuation of sickness behavior after the infectious/inflammatory stimulus ceased to exist. Hyperactivity of the CRH/HPA-axis can also be exacerbated by negative emotions that can act as stressors and further propagate the symptoms of sickness behavior, with the potential to develop stress-related disorders. In fact, chronic depressive moods themselves may lead to cortisol-induced atrophy of hippocampal neurons with suppressed negative feedback [50]. In the face of the important role of glucocorticoids in the negative feedback mechanism, any factor that leads to, or increases glucocorticoid-resistance could significantly enhance the possibility of the sickness behavior-associated neurobehavioral and proinflammatory activity to continue after cessation of the initiating disturbance. Although some pre- and post-receptor mechanisms may be involved, glucocorticoid resistance is mostly a function of alterations in receptor expression and function. Some of the underlying mechanisms have been reviewed by Miller et al. [52]. One mechanism very relevant to the contribution of cortisol resistance to the non-termination of the symptoms of sickness behavior is the role of cytokines. Cortisol resistance due a suppressed negative feedback may be induced by the very same cytokines that form part of the sickness behavior complex. Several studies showed that cytokines, especially those involved in inflammatory conditions, could alter glucocorticoid receptor density and functioning. Some contradictions exist between the results of those studies using whole cell techniques and those using cytosolic radioligand binding [52]. The overriding conclusion is, however, that pro-inflammatory cytokines can reduce the expression of glucocorticoid receptors as well as downregulate their functioning [52]. It is feasible to expect that this would suppress the negative feedback on the HPA-axis as well as cortisol's effects on cytokine production, resulting in continued activation of the stress axes and augmentation of pro-inflammatory cytokine production. This should in theory, via the proposed cerebral cytokine relay system [9], lead to a further increase in the activation state of the neuroendocrine stress axes - resulting in a type of positive feedback system that supports the inappropriate continuation of sickness behavior with the potential to progress towards behavioral disturbances.

Although genetic abnormalities in the glucocorticoid receptor gene [53], with subsequent cortisol resistance, and polymorphism in glucocorticoid receptor genes with abnormal HPA-axis, regulation [54] exist, the magnitude of the contribution of genetics to glucocortioid resistance is not known. It is, however obvious that genetic alterations or failure of any factor involved in terminating the inflammatory process can contribute to the continuation of the sickness behavior. It is thus highly possible that individuals who are resistant to the effects of the feedback mechanisms, either through psychological predisposing or through the combination of genetics and predisposing and who subsequently develop sickness behavior may be at risk for non-termination of the sickness behavioral response and are thus at risk for the development of psychological disorders. Various factors, psychological and physiological, could influence the eventual outcome. Negative emotions appear to be amongst the most important contributors to the neurological and immunological dysregulation [1] and [55]. 


\section{Mental disorders as a continuation of sickness behavior}

For the normal homeostatic process to become a mental disturbance there must be similarities between the behavioral symptoms, the neuroendocrine disturbances and the cytokine profile. In this section the similarities between sickness behavior and a number of mental disorders are briefly discussed in support of the proposed hypothesis.

\section{Mood disorders and sickness behavior}

The similarities between the behavioral characteristics of the mood disorders and that of sickness behavior are striking. As previously seen, sickness behavior symptoms may vary with the severity and duration of the condition from flu-like symptoms to anhedonia to severe cognitive disturbances. In considering the symptoms of mood disorders (depressed mood most of the day; nearly every day; markedly diminished interest or pleasure in almost all activities; decreased appetite or weight loss, hypersomnia or insomnia nearly every day; psychomotor retardation or agitation nearly every day; fatigue or loss of energy nearly every day; feelings of worthlessness or excessive or inappropriate guilt; diminished ability to think or concentrate and recurrent thoughts of death, suicidal ideation or a suicide attempt - from which at least 5 have to be present to represent a major depressive episode) [56], it is clear that they all fall within the scope of the symptoms of sickness behavior.

For quite a while, there have been indications that the immune system may play a causal role in depressive moods, specifically in the etiology of major depression. Major milestones in the development of the hypothesis that immune changes may underlie the etiology of major depression include the macrophage theory of depression by Smith, 1991 [57], followed by the interleukin hypothesis of major depression as described by Maes in 1995 [58]. Direct evidence for the presence of an inflammatory response in mood disorders comprises findings such as increases in circulating monocytes and neutrophils, increased neopterin and prostaglandin levels, activation of the acute phase response, and increases in pro-inflammatory cytokines such as IL-1 $\beta$, IL-6 and IFN $\gamma$ [17]. Of interest is the fact that most of these changes can be induced by the same pro-inflammatory cytokines responsible for the symptoms of sickness behavior [6], [8] and [10].

There is a good correspondence between the basic neuroendocrine perturbations of sickness behavior and the major depressive disorder. The hypersecretory state of the HPA-axis of major depression [59] is, (as for sickness behavior) ascribed to hypersecretion of CRH due to, amongst other things, a suppression in the negative feedback mechanism - both exhibiting non-suppression of cortisol secretion upon dexamethasone administration - a form of cortisol resistance. The degree of non-suppression would appear to correlate with the levels of Il-1 $\beta$ [52], both in depression and sickness behavior. This is in agreement with findings that immune challenges could decrease the affinity of the corticosteroid receptors involved in the negative feedback of the HPA-axis and that it may be a factor in major depression [52] and [13]. Immune challenge-induced pro-inflammatory cytokines in sickness behavior can, in turn, stimulate CRH/HPA-axis activity [11] and thus cause hyperactivity of the CRH/HPA system. Immunological activity, as seen in sickness behavior can thus increase CRH activity both by stimulating the secretion directly and by inhibiting the negative feedback. Major depression is similarly associated with a central CRH drive as represented by above normal CRH levels in the cerebrospinal fluid, increased CRH mRNA levels in the paraventricular nucleus and reduced CRH receptor density in the frontal cortex [60]. This central CRH drive which is supposed to be of short duration during sickness behavior and which is present in a more chronic form with major depression, is said to act as a compensatory mechanism against the effects of the elevated cortisol levels on immune function. This may, however, be a potentially dangerous situation in conditions of glucocorticoid receptor downregulation where the glucocorticoid suppression of pro-inflammatory cytokines is reduced. Such a condition of proinflammatory cytokine stimulation by $\mathrm{CRH}$ and downregulation of the cortisol anti-inflammatory activity 
may lead to unbridled continuation of sickness behavior, or in the case of individuals with trauma or serious infections to the systemic inflammatory response syndrome, septic shock, or even multiple organ failure. In case of the first possibility, i.e., non-termination of sickness behavior, it is highly feasible to expect that the behavioral symptoms would reflect that of major depression. In fact, that it could indeed be a case of sickness behavior that developed into a chronic state of depression. It is clear how predisposition to lower activation threshold and suppressed negative feedback could render the individual vulnerable to non-termination and cause a switch from appropriate sickness behavior to a dysfunctional major depressive state.

The serotonin abnormalities associated with major depression [61] would appear to include, amongst other, central nervous system changes in presynaptic serotonergic neurons due to a deficiency in 1tryptophan availability. A similar decrease in tryptophan availability, due to a cytokine-induced shift to the kynurenine pathway, has been reported for sickness behavior [10]. At this stage it would be risky to venture into an in depth comparison between the alterations in serotonin metabolism as it is becoming clear that the various cerebral areas undergo different changes in their serotonin metabolism and receptor activity - both during immunologically induced sickness behavior and with major depression [60].

If sickness behavior can evolve into mood disorders such as major depression or the dysthymic disorder it should in theory be possible that the factors responsible for the neurological disturbances of sickness behavior, i.e., pro-inflammatory cytokines, should also be able to lead to the neurological disturbances of depression. This is indeed the case. Hyperactivity of the HPA-axis can, for instance be induced by Il-1 and Il-6 in cooperation with other cytokines, by inflammatory-related changes in noradrenaline and serotonin turnover, and by any factor that causes a downregulation of the negative feedback of the HPAaxis [11]. In addition, noradrenergic hyperactivity can be induced by cytokines, either directly, or as a result of the control of the CRH/HPA-axis over the system and the positive reverberating influence between the two stress axes [12] and [62]. Central serotonin is also known to be influenced by the proinflammatory reaction seen in sickness behavior. Pro-inflammatory cytokines such as Il-1 $\beta$, IFN $\gamma$ and $\mathrm{TNF} \alpha$ have, for instance, been shown (a) to alter extracellular serotonin (5HT) levels in the hypothalamus, the hippocampus and the cortex, (b) to modulate the activity of the serotonin transporter involved in central serotonergic neurotransmission by serotonin re-uptake, and (c) to induce the activity of the first enzyme of the kynurenine pathway (indoleamine-2,3-dioxygenase) that converts tryptophan to kynurenic acid and quinolinic acid with a resultant tryptophan-depletion and/or reduction in the tryptophan to competing amino acid ratio with the potential for a reduced serotonin production [Reviewed by Maes, 1999 [17]].

\section{Depression due to a general medical condition}

Depression due to a general medical condition is often seen merely as a psychological reaction to the disability, pain and loss of functionality. However, empirical evidence suggests that the depression and several other cognitive symptoms found in such conditions are causally related to pro-inflammatory activity rather than mere expressions of a psychological reaction to the medical condition [13]. Many medical conditions, including almost all chronic and some acute infectious and inflammatory conditions, as well as trauma and surgery - in fact virtually any form of tissue injury, are associated with the typical neuroimmunological profile of sickness behavior, which comprises activation of the inflammatory response accompanied by alterations in the CRH/HPA-axis, CNA/SAM-axis, and central serotonergic activity. Amongst the non-infectious medical conditions where hyperactivation and secretion of cytokines have been reported with the highest incidence are various autoimmune diseases, allergies, multiple sclerosis, rheumatoid arthritis, stroke, trauma, the premenstrual syndrome, post partum depression [13], several neurodegenerative disorders, sepsis and the systemic inflammatory response syndrome. The condition of resistance to termination can be exacerbated or prolonged by negative perceptions, feelings of no control or the inability to cope - in fact, by any factor that causes psychological stress [1] and [63]. 
The fact that antidepressants would appear to alleviate the depressive symptoms does not argue against a causal role for pro-inflammatory cytokines as these substances are reported to suppress the production of pro-inflammatory cytokines and to alleviate pain [13] and [64]. It is thus clear that, although the depression often found in association with medical conditions may be the expression of sickness behavior, it does present with the possibility to develop into a chronic mood disorder - especially in individuals predisposed to it by their genetic make up and early life experiences and that negative emotions increase the possibility. In view of the variety of behavioral symptoms, ranging from mild constitutional to psychosis that may be induced by pro-inflammatory cytokines, one should perhaps rather refer to behavioral disturbances due to a general medical condition, rather than depression due to a general medical condition.

\section{Other mental disorders}

In addition to be the stimulus for the expression of mood disorders, sickness behavior could very well also serve as the trigger for the development of other mental disorders to which the individual has been predisposed. This statement is based on two facts. The first is that pro-inflammatory cytokines, the initiating factors of the neurobehavioral adaptations of sickness behavior, can lead to a wide spectrum of behavioral problems, including severe chronic fatigue, neurasthenia, asthenia, malaise, lethargy, somnolence, emotional instability, crying spells, agitation, irritability, short temper, overreaction to problems and frustrations, dementia, hallucinations, delirium, delusional ideation, impaired memory and amnesia, aphasia, clouding of consciousness, confusion, disorientation, personality changes, seizures, coma, increased sensitivity to and inability to cope with pain, Parkinson's like symptoms, anorexia, psychomotor retardation, social withdrawal, depression and gesticulation [1], [12] and [23]. The second is the fact that pro-inflammatory activity has been reported for many mental disturbances. Examples of disturbances where abnormal cytokine activity have been reported include the panic disorder, generalized anxiety disorder, post-traumatic stress disorder, anorexia nervosa, obsessive-compulsive disorder, Sydenham's chorea, Tourette's syndrome, multiple sclerosis, Alzheimer's diseases, mood disorders, schizophrenia, other psychotic disorders and many others [63], [65], [66], [67] and [68]. The presence of cytokine, especially pro-inflammatory cytokine, disturbances in various different mental disorders confirms the fact that specific patterns of immune alterations can not summarily be seen as correlates of specific behavioral disturbances. The stimulatory effects of cytokines on the CRH/HPA-axis are fairly well known but a more in depth account can be found in a review by Buckingham et al., [11]. Other effects of individual cytokines which could have a bearing on psychopathology have been reviewed by Muller and Ackenheil, 1998 [12] including indications that (a) Il-1, with high receptor density in the hippocampus, hypothalamus and brain stem, stimulates the release of peripheral catecholamines as well as brain stem and hypothalamic noradrenaline, and increases the turnover of noradrenaline, serotonin, tryptophan and dopamine in the brain, (b) Il-2, with high receptor density in the locus coeruleus and pyramidal cell layer of the hippocampus, stimulates dopaminergic neurotransmission - particularly dopaminergic metabolism in the prefrontal cortex and may exert an effect on motor function by regulating striatal dopaminergic functions. It also increases hippocampal noradrenergic metabolism and may have a sedative effect through its influence on the locus coeruleus and nucleus caudatus. It has further been shown to inhibit acetylcholine release in the hippocampus and frontal cortex and may lead to neuronal loss and degenerative changes in the hippocampus with loss of mnestic functions, (c) Il-6, with its high receptor density in the hippocampus and prefrontal cortex, stimulates the secretion of dopamine and noradrenaline and increases dopamine and serotonin turnover in the hippocampus and prefrontal cortex, (d) TNF- $\alpha$ would appear to have a biphasic influence with stimulatory effects on the catecholaminergic systems in the acute situation and suppressive effects upon chronic exposure. It has further been implicated in cytotoxicity and demyelinization. Most of the above effects reflect that of single cytokines in the acute situation and we are still far from understanding the synergistic interaction between the various pro-inflammatory cytokines on cerebral structure and function. By implication it 
would therefore be possible that non-termination of the inflammatory response of sickness behavior can precipitate any one of a number of mental disorders to which the individual has been predisposed.

\section{Sickness behavior and disorders associated by low cortisol}

In addition to hyperactivation, early life experiences may also predispose to hypoactivity of the $\mathrm{CRH} / \mathrm{HPA}$-axis. A fact that, at first glance, would appear to directly predispose to inappropriate continuation of the inflammatory response - even in the absence of cortisol resistance. In fact, low cortisol levels may even stimulate the secretion of pro-inflammatory cytokines [49]. In contrast to the predisposition to hyperactivity of the CRH/HPA-axis, our knowledge on the physiological aspects of those mental disorders where hypoactivity of the axis has been reported is rather scant and results often contradictory. Hypocortisolaemia, often accompanied by baseline sympathetic nervous system hypofunction and low serum serotonin and L-tryptophan levels, is reported for subsets of patients with PTSD, fibromyalgia, chronic fatigue syndrome, chemical intolerance and other stress related somatic disorders [59], [69] and [70]. Amongst the disorders where HPA-axis hypoactivity can occur, PTSD differs from the others, not only in its etiology, but also at the level where the suppression occurs. PTSD is a typical severe stress-related disturbance, classified according to the DSM-IV as an anxiety disorder [71]. In contrast to many other stressor-induced disorders, the precipitating traumatic event can usually be precisely identified, and re-experiencing of the event may constitute a major contribution to nonextinction and exacerbation of the allostatic load. Early life experience, genetics and support systems are bound to play a role in the development and progression as distinct differences exist between the psychological symptoms and neurological profiles of patients. PTSD would appear to be characterized by CRH hypersecretion with subsets of low and subsets of normal to high cortisol secretion [59], [72], [73] and [74]. A feasible suggestion on the CRH/HPA-axis disturbance in PTSD would appear to be that of Ehlert and Heinrichs, 2001, [59], i.e., that PTSD is associated with alterations of the axis that can be interpreted as a latent hypocortisolism with an increase in the feedback inhibition to the pituitary and the adrenals, while neuronal CRH release appears to be above normal. A possible alternative explanation is that the activity of the HPA-axis in PTSD may change over time [75], depending on the development and progression of the disease which in turn could be expected to be a function of priming by early life experiences, of genetics, and in general on the presence of vulnerability and resilience factors [1]. It is tempting to speculate that the relative adrenal insufficiency in PTSD could represent adrenal burn-out due to uncontrolled stress-induced stimulation during the earlier stages, and that the hypersecretion of $\mathrm{CRH}$ be due to the reduced feedback and perhaps cortisol-induced hippocampal damage at the stage of trauma or during the initial stages of development of PTSD. A degree of hippocampal atrophy has indeed been observed in combat veterans with PTSD [76]. It is thus essential that future research take in to consideration the stage as well as the symptoms. There is evidence that pro-inflammatory cytokines may contribute to the anhedonia and anxiety of PTSD [77] and [78].

In addition to PTSD, low cortisol levels have also been reported in somatoform disorders and related syndromes. Controversy still exists as to whether syndromes consisting of subjective health complaints represent several or variations on one general condition. In fact, it has been suggested that most of the multisymptom syndromes like somatoform disorders, fibromyalgia, chronic fatigue syndrome and exposure syndromes such as Gulf War illnesses, sick-building syndrome, illnesses found in women with silicon breast implants, multiple chemical sensitivity and others are related in terms of their stressexposure histories, their psychological profiles, and some neurological alterations [69]. No distinction will therefore be made for the purpose of this discussion. According to the DSM-IV definition [79], somatoform disorders can be defined as the presence of physical symptoms that suggest a general medical condition, but are not fully explained by a medical condition, by the direct effects of a substance, or by another medical disorder. Idiopathic pain, fatigue and gastrointestinal disturbances form important aspects, not only of several of the somatoform disorders/multisymptom syndromes where the symptoms cannot be fully ascribed to a specific medical condition. Low cortisol levels have been reported in various 
types of idiopathic pain and with gastrointestinal disorders for which no organic cause could be found. Typical examples of hypocortisolism in association with idiopathic pain include recurrent headaches, idiopathic chronic pelvic pain in women, recurrent abdominal pain in children and idiopathic pain in combination with functional gastrointestinal disorders. In most of these patients, where low cortisol levels were found, there were also histories of poor coping abilities, multiple major stressful life events, and in women with chronic pelvic pain, high rates of physical and sexual abuse. In fact it would appear that most individuals with serious multiple unexplicable health complaints have previously suffered from clusters of severe infectious complications, physical or emotional trauma [69] - factors earlier in this paper seen to have the ability to predispose to hypofunction of the CRH/HPA-axis and to stimulate the production of pro-inflammatory cytokines. Preliminary indications are that adrenal insufficiency and an above normal sensitivity to the negative feedback may underlie the hypofunction of the HPA-axis [59]. To accept that sickness behavior can develop into syndromes and disorders marked by multiple subjective health complaints, such as with fibromyalgia, chronic fatigue syndrome or Gulf War Syndrome one would expect to find indications of pro-inflammatory activity. There are reasons to suspect that an inflammatory process may be present in those subsets of individuals characterized by hypoactivity of the HPA-axis. The first and most obvious reason is that of a deficiency of cortisol - one of the most potent inhibitors of inflammation. In addition, it is known that Il-6 levels can be associated with chronic distress and feelings of uncontrollability [80]. It is also known that cytokines can stimulate inflammatory pain and that Il-1 per se may lead to an increase in pain sensitivity in general [81]. Some authors reported increases in most of the pro-inflammatory cytokines (Il-1, Il-6, TNF- $\alpha$ ) [70], [82], [83] and [84] but there are still many discrepancies in results. The picture with regard to the inflammatory cytokines in disorders with multiple subjective health complaints is, however, far from clear. Nevertheless, even without proper empirical information on the pro-inflammatory status, there are reasons, such as the similarity in symptoms, the apparent history of factors such as infectious or traumatic events, and the suppressed negative feedback on inflammatory cytokine production as a result of low cortisol, to suspect that sickness behavior may render the individual vulnerable to the development of somatoform and related multi-symptom or subjective health complaint disturbances. In the case of especially somatoform-related disturbances it is important to take note of the possibility that sickness behavior may become a conditioned response [6].

\section{Summary}

This paper introduces the concept of non-termination of sickness behavior as a potential threat to mental health. In view of the similarities between the symptoms, the neurohormonal changes and the cytokine profiles of sickness behavior and that of a number of mental disorders it is hypothesized that the inappropriate continuation of sickness behavior, (i.e., non-termination), after recovery from the initial disease, could form the basis for mental disturbances. This would be particularly relevant in individuals with alterations in stress vulnerability, which may occur due to the combination of genetic disposition and priming by early life experiences. The non-termination of sickness behavior may thus precipitate a state of psychopathology associated with hypersecretion of pro-inflammatory cytokines, either through cortisol resistance in the case of mental disturbances marked by hyperactivation of the stress axes or through hypocortisolaemia. The coordinated set of behavioral and other changes that constitute sickness behavior represents a new homeostasis or state of adaptation intended to aid in coping with whatever stressor threatens the primary homeostasis. It is therefore merely the non-termination of the adaptation process that determines the transition of sickness behavior, as process of coping and adaptation, to a state of psychopathology with related symptoms and not necessarily the development of a maladaptive variant of the adaptive response. Negative emotions can, by stimulating the production of pro-inflammatory cytokines, significantly increase the potential for non-termination. The concept of non-termination of sickness behavior as precipitating factor for the development of mental disorders is summarized in Fig. 2. 


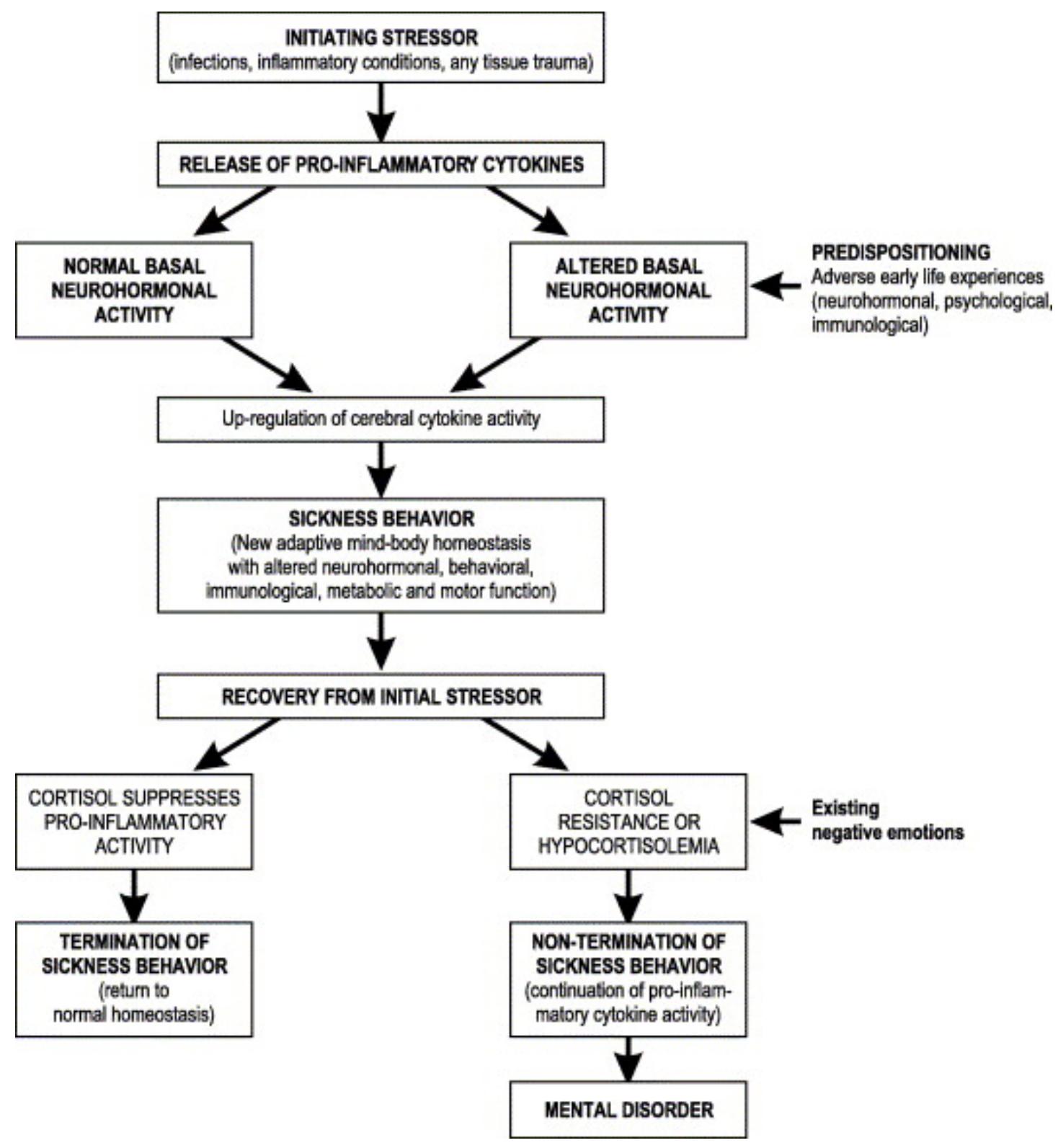

Figure 2. Non-termination of sickness behavior. Infections, inflammation and trauma can initiate the development of sickness behavior through stimulation of the release of pro-inflammatory cytokines. Sickness behavior is normally terminated when the individual recovers from the initiating stressor, i.e., the physical disease. Adverse early life experiences may predispose an individual to non-termination of sickness behavior by either hyper- or hyporesponsivity of the CRH/HPA-axis, leading to cortisol resistance or hypocortisolemia, respectively. Such individuals would be at risk to continue with unopposed proinflammatory cytokine activity even after recovery from the initiating stressor. Increased pro-inflammatory cytokine activity is known to be present in many psychiatric disorders, and may be important in the etiology of these diseases.

This psychoneuroimmunological approach to mental disease supports the multiple entry point concept as suggested by the biopsychosocial approach [85]. It is obvious that the stimulatory input (presensitization) can be at, and the effector output (psychopathology) from, virtually any point in the behavioral, neuroendocrine or immunological spectra. Presensitization may even occur during the early neurodevelopmental period, not only as a result of an abnormal endocrinological milieu, but also as a result of immunological events, as cytokines are involved in processes such as neuronal development, synaptogenesis, regeneration and plasticity, and over- or underproduction of cytokines may alter the development and responsiveness of the central nervous system [86]. 
Several aspects need further investigation in order to come to a clearer understanding on the proposed transition from sickness behavior to psychopathology. It seems necessary that researchers should subgroup their patients according to their HPA-axis and sympathetic nervous system activity when examining the immunological status and that the medical and psychosocial history, as well as psychiatric symptoms be considered. It should be kept in mind that sickness behavior is in part a motivation state in competition with other motivation states where the behavioral output can be altered according to circumstances and that it could probably even become a conditioned response. The non-termination of pro-inflammatory cytokine production and sickness behavior symptoms therefore may become, within limits, a function of the individual's perceptions and emotional needs. It is also known that crosssensitization can occur between stressors and that emotional states can influence the neurobehavioral and immunological aspects of sickness behavior. Therapeutic interventions should take these aspects into consideration.

\section{References}

[1] J.K. Kiecolt-Glaser, L. McGuire, T.F. Robles and R. Glaser, Emotions, morbidity, and mortality. New perspectives from psychoneuroimmunology, Ann Rev Psychol 53 (2002), pp. 83-107.

[2] R. Dantzer, Cytokine-induced sickness behaviour: Where do we stand?, Brain Behav Immun 15 (2001) (1), pp. 7-24.

[3] R. Dantzer, R.M. Bluthe, N. Castanon, N. Chauvet, L. Capuron and G. Goodall et al., Cytokine effects of behaviour (3rd ed.). In: R. Ader, D.L. Felten and N. Cohen, Editors, Psychoneuroimmunology vol. 1, San Diego, Academic Press (2001), pp. 703-725.

[4] B.G. Charlton, The malaise theory of depression: major depressive disorder is sickness behavior and antidepressants are analgesics, Med Hypoth 54 (2000) (1), pp. 126-130

[5] N.E. Miller, Some psychophysiological studies of motivation and of the behavioural effects of illness, Bull Br Psychol 17 (1964), pp. 1-20.

[6] R. Dantzer, Cytokine-induced sickness behavior: mechanisms and implications, Ann NY Acad Sci 933 (2001), pp. 222-234

[7] A. Panzer, M. Viljoen, R. Maunder and M. Hunter, Difficult patients: the attachment perspective, SA Fam Pract 45 (2003) (8), pp. 11-13.

[8] R. Dantzer, A. Aubert, R.M. Bluthe, G. Gheusi, S. Cremona and S. Laye et al., Mechanisms of the behavioral effect of cytokines. In: R. Dantzer, E.E. Wollman and R. Yirmiya, Editors, Cytokines, stress and depression. Advances in experimental medicine and biology vol. 461, Kluwer Academic/Plenum Publishers, New York (1999), pp. 83-105

[9] H.O. Besedovsky and A. Del Rey, Cytokines as mediators of central and peripheral immuneneuroendocrine interactions (3rd ed.). In: R. Ader, D.L. Felton and N. Cohen, Editors, Psychoneuroimmunology vol. 1, Academic Press, San Diego (2001), pp. 1-17.

[10] J.P. Konsman, P. Parnet and R. Dantzer, Cytokine-induced sickness behavior: mechanisms and implications, Trends Neurosci 25 (2002) (3), pp. 154-160. 
[11] J.C. Buckingham, H.D. Loxley, H.C. Christian and J.G. Philip, Activation of the HPA axis by immune insults: roles and interactions of cytokines, eicosanoids, and glucocorticoids, Pharmacol Biochem Behav 54 (1996), pp. 285-288.

[12] M. Muller and M. Ackenheil, Psychoneuroimmunology and the cytokine action in the CNS: implication for psychiatric disorders, Prog Neuropsychopharmacol Biol 22 (1998), pp. 1-33.

[13] R. Yirmiya, J. Weidenfeld, Y. Pollak, M. Morag, A. Morag and R. Avitsur et al., Cytokines, 'depression due to a general medical condition', and antidepressant drugs. In: R. Dantzer, R. Wollman and R. Yirmiya, Editors, Cytokines, stress and depression. Advances in experimental medicine and biology vol. 461, Kluwer Academic/Plenum Publishers, New York (1999), pp. 283-316

[14] M. Viljoen and A. Panzer, Sickness behaviour, SA Fam Pract 45 (2003) (9), pp. 15-18.

[15] A.C.E. Linthorst and J.M.H.M. Reul, Brain neurotransmission during peripheral inflammation, N Y Acad Sci 840 (1998), pp. 139-152

[16] A.J. Dunn, J. Wang and T. Ando, Effects of cytokines on cerebral neurotransmission. Comparison with the effects of stress. In: R. Dantzer, E.E. Wollman and R. Yirmiya, Editors, Cytokines, stress and depression. Advances in experimental medicine and biology vol. 461, Kluwer Academic/Plenum Publishers, New York (1999), pp. 117-127

[17] M. Maes, Major depression and activation of the inflammatory response system. In: R. Dantzer, E.E. Wollman and R. Yirmiya, Editors, Cytokines, stress and depression. Advances in experimental medicine and biology vol. 461, Kluwer Academic/Plenum Publishers, New York (1999), pp. 25-46.

[18] M.H. Rapaport and N. Muller, Immunological states associated with schizophrenia (3rd ed.). In: R. Ader, D.L. Felton and N. Cohen, Editors, Psychoneuroimmunology vol. 1, Academic Press, San Diego (2001), pp. 373-382.

[19] F. Marx, I. Blasko, M. Pavelka and B. Grubeck-Loebenstein, The possible role of the immune system in Alzheimer's disease, Exp Geront 33 (1998) (7-8), pp. 871-881

[20] P. Schubert and K. Rudolphi, Interfering with the pathological activation of microglial cells and astrocytes in dementia, Alz Dis Ass Dis 12 (1998) (Suppl. 2), pp. S21-S28

[21] B.B. Mittleman, F.X. Castellanos, L.K. Jacobsen, J.L. Rapoport, S.E. Swedo and G.M. Shearer, Cerebrospinal fluid cytokines in pediatric neuropsychiatric disease, J Immunol 159 (1997) (6), pp. 29942999

[22] H. Anisman and Z. Merali, Anhedonic and anxiogenic effects of cytokine exposure, Adv Exp Med Biol 461 (1999), pp. 199-233.

[23] R.C. Turowski and P.L. Triozzi, Central nervous system toxicities and cytokine therapy. In: N.P. Plotnikoff, R.E. Faith, A.J. Murgo and R.A. Good, Editors, Cytokines stress and immunity, CRC Press, Boca Raton (1999), pp. 93-114.

[24] F.J.H. Tilders and E.D. Schmidt, Cross-sensitization between immune and non-immune stressors. A role in the etiology of depression?. In: R. Dantzer, E.E. Wollman and R. Yirmiya, Editors, Cytokines, stress and depression. Advances in experimental medicine and biology vol. 461, Kluwer Academic/Plenum Publishers, New York (1999), pp. 179-197. 
[25] A. Panzer and M. Viljoen, Associations between psychological profiles and diseases: examining hemispheric dominance and autonomic activation as underlying regulators, Med Hypoth 61 (2003) (1), pp. 75-79

[26] D.D. Francis, F.C. Caldji, F. Champagne, P.M. Plotsky and M.J. Meany, The role of corticotropinreleasing factor-norepinephrine systems in mediating the effects of early experience on the development of behavioural and endocrine responses to stress, Soc Biol Psychiatry 46 (1999), pp. 1153-1166.

[27] C. Caldji, J. Diorio and J. Meaney, Variations in maternal care regulate the development of stress reactivity, Biol Psychiatry 48 (2000), pp. 1164-1174

[28] K. Pacak, M. Palkovits, I. Kopin and D.S. Goldstein, Stress-induced norepinephrine release in hypothalamic paraventricular nucleus and pituitary adrenocortical and sympathoadrenal activity: in vivo microdialysis studies, Front Neuroendocrinol 16 (1995), pp. 89-150

[29] C.-D. Walker, D.J. Toufexis and A. Burlet, Hypothalamic and limbic expression of CRH and vasopressin during lactation: implications for the control of ACTH secretion and stress hyporesponsiveness, Prog Brain Res 133 (2001), pp. 99-110

[30] M. Altemus, P.A. Deuster, E. Galliven, C.S. Carter and P.W. Gold, Suppression of hypothalamicpituitary-adrenal axis responses to stress in lactating mothers, J Clin Endocrinol Metab 80 (1995), pp. 2954-2959.

[31] J. Schulkin, P.W. Gold and B.S. McEwen, Induction of corticotropin-releasing hormone gene expression by glucocorticoids: implication for understanding the states of fear, anxiety and allostatic load, Psychoneuroendocrinology 23 (1998) (3), pp. 219-243

[32] D.D. Francis and M.J. Meany, Maternal care and the development of stress responses, Curr Op Neurobiol 9 (1999) (1), pp. 128-134.

[33] M.J. Meany, J. Diorio, D. Francis, S. Weaver, J. Yau and K. Chapman, Postnatal handling increases the expression of cAMP-inducible transcription factors in the rat hippocampus: The effects of thyroid hormones and serotonin, J Neurosci 20 (2000), pp. 3395-3926.

[34] L. Arborelius, M.J. Owens, P.M. Plotsky and C.B. Nemeroff, CRF, depression and anxiety, J Endocrinol 160 (1999), pp. 1-12

[35] C.D. Ingram, G. Fairchild, D.A. Johnson, C. Troakes, C. Lowry and S.L. Lightman et al., CRH and glucocorticoid actions on raphe serotonergic neurons, Stress 5 (2002) (Suppl.), p. 24.

[36] S.G. Matthews, D. Owen, S. Banjanin and M.H. Andrews, Glucocorticoids, endocrine development and life after birth, Stress 5 (2002) (Suppl.), p. 26.

[37] S.L. Cummings, W.S. Young and J.S. King, Early development of cerebellar afferent systems that contain corticotropin-releasing factor, J Comp Neurol 350 (1994) (4), pp. 534-549

[38] R.E. Grunau, Early repetitive stress in preterm infants: effects on behaviour and development, Stress 5 (2002) (suppl), p. 27. 
[39] L. Calza, L. Giardino, M. Pozza, A. Micera and L. Aloe, Time-course changes in nerve growth factor, corticotropin-releasing hormone and nitric oxide synthase isoforms and their possible role in the development of inflammatory response in experimental allergic encephalomyelitis, Prog Nat Acad Sci USA 94 (1997) (7), pp. 3368-3373.

[40] J.M. Worlein and M.L. Laudenschlager, Effects of early rearing experiences and social interactions on immune function in non-human primates (3rd ed.). In: R. Ader, D.L. Felton and N. Cohen, Editors, Psychoneuroimmunology vol. 1, Academic Press, San Diego (2001), pp. 73-85.

[41] V.P. Bakshi and N.H. Kalin, Corticotropin-releasing hormone and animal models of anxiety: geneenvironmental interactions, Biol Psychiatry 48 (2000), pp. 1175-1198

[42] C. Heim, Neurobiological correlates of early adverse experience in women, Stress 5 (2002) (Suppl.), p. 28.

[43] A. Armario, A single exposure to a severe stressor causes long-term desensitization of the physiological response to the homotypic stressor, Stress 5 (2002) (Suppl.), p. 16.

[44] M.M. Sanchez and K.J.S. Anand, Long-term consequences of early painful experiences, Stress 5 (2002) (Suppl.), p. 17.

[45] D.I.W. Phillips, Fetal growth, the HPA axis and the metabolic syndrome, Stress 5 (2002) (Suppl.), p. 28.

[46] I.J. Ellenkov, R.L. Wilder, G.P. Chrousos and E.S. Vizi, The sympathetic nerve - An integrative interface between two super systems: the brain and the immune system, Pharmacol Rev 52 (2000), pp. 595-638.

[47] I.J. Ellenkov, E.L. Webster, D.J. Torpy and G.P. Chrousos, Stress, corticotropin-releasing hormone, glucocorticoids and the immune/inflammatory response, Ann N Y Acad Sci 876 (1999), pp. 1-13.

[48] J.P. Herman, C.M.F. Prewitt and E. Cullinan, Neuronal circuit regulation of the hypothalamopituitary-adrenal stress axis, Crit Rev Neurobiol 10 (1996) (3\&4), pp. 371-394.

[49] L.R. Watkins, K.T. Nguyen, J.E. Lee and S.F. Maier, Dynamic regulation of proinflammatory cytokines. In: R. Dantzer, E.E. Wollman and R. Yirmiya, Editors, Cytokines, stress and depression. Advances in experimental medicine and biology vol. 461, Kluwer Academic/Plenum Publishers, New York (1999), pp. 153-178.

[50] Y.I. Sheline, P.W. Wang, M.K. Gado, J.G. Csernansky and W.W. Vannier, Hippocampal atrophy in recurrent major depression, Proc Natl Acad Sci 93 (1996), pp. 3908-3913

[51] J. Raber, Detrimental effects of chronic hypothalamic-pituitary-adrenal axis activation. From obesity to memory deficits, Mol Neurobiol 18 (1998), pp. 1-22.

[52] A.H. Miller, C.M. Pariante and B.D. Pearce, Effects of cytokines on glucocorticoid receptor expression and function. Glucocorticoid resistance and relevance to depression. In: R. Dantzer, R. Wollman and R. Yirmiya, Editors, Cytokines, stress and depression. Advances in experimental medicine and biology vol. 461, Kluwer Academic/Plenum Publishers, New York (1999), pp. 107-116 
[53] M. Ruiz, U. Lind, M. Gafvels, G. Eggertsen and L. Nilsson, Characteristics of two novel mutations in the glucocorticoid receptor gene in patients with primary cortisol resistance, Clin Endocrinol 55 (2001) (3), pp. 363-371

[54] P. Bjorntorp and R. Rosmond, The metabolic syndrome - a neuroendocrine disorder?, Brit J Nutr 83 (2000) (Suppl. 1), pp. S49-S57

[55] J.K. Kiecolt-Glaser, L. McGuire, T.F. Robles and R. Glaser, Psychoneuroimmunology and psychosomatic medicine: back to the future, Psychosom Med 64 (2002), pp. 15-28.

[56] Diagnostic and Statistical Manual of Mental Disorders. 4th ed. Washington (DC): American Psychiatric Association; 1994. p. 317-89.

[57] R.S. Smith, The macrophage theory of depression, Med Hypoth 35 (1991), pp. 298-305.

[58] M. Maes, The interleukin hypothesis of major depression, Prog Neuropsychopharmacol Biol Psych 19 (1995), pp. 11-35.

[59] U. Ehlert and J.G.M. Heinrichs, Psychoneuroendocrinological contributions to the etiology of depression, posttraumatic stress disorder, and stress-related bodily disorders: the role of the hypothalamus-pituitary-adrenal axis, Biol Psychol 57 (2001), pp. 141-152.

[60] A.C.E. Lindhorst and J.M.H.M. Reul, Inflammation and brain function under basal conditions and during long-term elevation of brain corticotropin-releasing hormone levels. In: R. Dantzer, R. Wollman and R. Yirmiya, Editors, Cytokines, stress and depression. Advances in experimental medicine and biology vol. 461, Kluwer Academic/Plenum Publishers, New York (1999), pp. 129-152.

[61] M. Maes and H.Y.M. Meltzer, The serotonin hypothesis of major depression. In: F. Bloom and D. Kupher, Editors, Psychopharmacology: the fourth generation of progress, Raven Press, New York (1995), pp. 933-944.

[62] G.P. Chrousos, Stressors, stress and neuroendocrine integration of the adaptive response, NY Acad Sci 851 (1998), pp. 311-355.

[63] M. Maes, C. Song, A. Lin, R.D. Jongh, A. Van Gastel and G. Kenis et al., The effects of psychological stress on humans: increased production of proinflammatory cytokines and a Th-1 like response in stress-induced anxiety, Cytokine 10 (1998), pp. 313-318

[64] B.G. Charlton, The malaise theory of depression: major depressive disorder is sickness behavior and antidepressants are analgesics, Med Hypoth 54 (2000) (1), pp. 126-130

[65] J.E. Merrill, Production and influence of inflammatory cytokines in diseases of the adult central nervous system (3rd ed.). In: R. Ader, D.L. Felten and N. Cohen, Editors, Psychoneuroimmunology vol. 1, Academic Press, San Diego (2001), pp. 547-561.

[66] B. Zhoa and J.P. Schwartz, Involvement of cytokines in normal CNS development and neurological disease: Recent progress and perspectives, J Neurosci Res 52 (1998), pp. 7-16.

[67] R. Weizman and H. Bessler, Cytokines: stress and immunity - an overview. In: N.P. Plotnikoff, R.E. Faith, A.J. Murgo and R.A. Good, Editors, Cytokines stress and immunity, CRC Press, Boca Raton (1999), pp. 1-15. 
[68] N. Muller and M. Ackenheil, The immune system and schizophrenia. In: B.E. Leonard and K. Miller, Editors, Stress, the immune system and psychiatry, Wiley and Sons, Chichester (1995), pp. 137164.

[69] D.J. Clauw, Potential mechanisms in chemical intolerance and related conditions, Ann NY Acad Sci 933 (2001), pp. 235-253

[70] J.T.J. Visser, E.R. De Kloet and L. Nagelkerken, Altered glucocorticoid regulation of the immune response in chronic fatigue syndrome, Ann NY Acad Sci 917 (2000), pp. 868-875

[71] Diagnostic and Statistical Manual of Mental Disorders. 4th ed. Washington (DC): American Psychiatric Association; 1994. p. 424.

[72] D.G. Baker, S.A. West, W.E. Nicholson, N.N. Ekhator, J.W. Kasckow and K.K. Hill et al., Serial corticotropin-releasing hormone levels and adrenal cortical activity in combat veterans with posttraumatic stress disorder, Am J Psych 156 (1999), pp. 585-588

[73] M.B. Stein, R. Yehuda, C. Koverola and C. Hanna, Enhanced dexamethasone suppression of plasma cortisol in adult women traumatized by childhood abuse, Biol Psychiatry 42 (1997), pp. 680-686.

[74] A.K. Goenjian, R. Yehuda, R.S. Pynoos, A.M. Steinberg, M. Tashjian and M. Yang et al., Basal cortisol, dexamethasone suppression of cortisol and MHPG in adolescents after the 1988 earthquake in Armenia, Am J Psychiatry 153 (1996), pp. 929-934

[75] S. Wang, J.P. Wilson and J.W. Mason, Stages of decompensation in combat-related posttraumatic stress disorder: a new conceptual model, Integ Physiol Behav 31 (1996), pp. 237-253

[76] T.V. Gurvits, M.E. Shenton, H. Hokama, H. Ohta, N.B. Lasko and M.W. Gilbertson et al., Magnetic resonance imaging study of hippocampal volume in chronic, combat-related posttraumatic stress disorder, Biol Psychiatry 40 (1996), pp. 1091-1099

[77] B. Spivak, B. Shohat, R. Mester, S. Avraham, I. Gil-Ad and A. Bleich et al., Elevated levels of serum interleukin-1 beta in combat-related posttraumatic stress disorder, Biol Psych 42 (1997) (5), pp. $345-348$

[78] H. Anisman and Z. Merali, Anhedonic and anxiogenic effects of cytokine exposure, Adv Exp Med Biol 461 (1999), pp. 199-233

[79] Diagnostic and Statistical Manual of Mental Disorders. 4th ed. Washington (DC): American Psychiatric Association; 1994. p. 445-69.

[80] W. Reif, F. Pilger, D. Ihle, E. Bosmans and M. Maes, Immunological differences between patients with major depression and somatization syndrome, Psychiatry Res 105 (2001), pp. 165-174.

[81] M. Schaefer and C. Stein, Control of pain in peripheral tissue by cytokines and neuropeptides. In: N.P. Plotnikoff, R.E. Faith, A.J. Murgo and R.A. Good, Editors, Cytokines, stress and immunity, CRC Press, Boca Raton (1999), pp. 261-270.

[82] S.E. Strauss, Chronic fatigue syndrome: point and counterpoint, J Infect Dis 170 (1994), pp. 1-6. 
[83] A.L. Komaroff, Chronic fatigue syndrome: an update, Ann Rev Med 49 (1998), pp. 1-13

[84] E. Ferguson and H.J. Cassaday, Theoretical accounts of Gulf War Syndrome: from environmental toxins to psychoneuroimmunology and neurodegeneration, Behav Neurol 13 (2001-2002) (3-4), pp. $133-147$.

[85] L. Temoshok, On attempting to articulate the biopsychosocial model: psychological-psychophysical homeostasis. In: H.S. Friedman, Editor, Personality and disease, Wiley, New York (1983), pp. 205-257.

[86] J.E. Merrill, Tumor necrosis factor alpha, interleukin 1 and related cytokines in related brain development: normal and pathological, Dev Neurosci 14 (1992), pp. 1-10. 